\title{
REGULAR EMBEDDINGS WHICH PRESERVE LATTICE STRUCTURE ${ }^{1}$
}

\author{
PETER CRAWLEY
}

A lattice $L$ is said to be regularly embedded in a complete lattice $K$ if there exists an isomorphic mapping of $L$ into $K$ which preserves all least upper and greatest lower bounds that exist in $L$. With the MacNeille completion by cuts every lattice can be regularly embedded in a complete lattice. And if the lattice is a Boolean algebra, then its completion by cuts is also a Boolean algebra. In general, however, this embedding does not preserve lattice structure; there are examples of distributive lattices whose completions by cuts are not even modular. On the other hand every lattice $L$ can be embedded in the complete lattice of its ideals which satisfies every identity that holds in $L$. This embedding preserves greatest lower bounds but does not preserve infinite least upper bounds.

Recently A. Horn has asked whether or not any distributive lattice can be regularly embedded in some complete distributive lattice. Here this question is answered in the negative. We show with an example that there exists a distributive lattice which cannot be regularly embedded in a complete modular lattice.

What appears to be most important in the example is the lack of certain continuity laws. If we impose such a law on a lattice, then a structure preserving regular embedding is possible.

Following [2] let us define a subset $A$ of a lattice $L$ to be a complete ideal if for every element $a \in L$ and every subset $S \subseteq A$ for which US exists in $L$ we have

$$
a \cap \cup S \in A \text {. }
$$

The complete ideals of $L$ then form a complete lattice $K(L)$ under set inclusion, and the mapping of $L$ onto the set of its principal ideals is a regular embedding of $L$ into $K(L)$. Let us further define a lattice $L$ to be conditionally upper continuous if for every directed set of elements $x_{\delta}(\delta \in \Delta)$ for which $U_{\delta \in \Delta} x_{\delta}$ exists in $L$ and every $a \in L$,

$$
a \cap \bigcup_{\delta \in \Delta} x_{\delta}=\bigcup_{\delta \in \Delta} a \cap x_{\delta} .
$$

We then have the following theorem. If a lattice $L$ is conditionally upper continuous, then $K(L)$ is upper continuous and every identity

Received by the editors August 28, 1961.

1 This work was supported by the Office of Naval Research. 
that holds in $L$ also holds in $K(L)$. It therefore follows that every conditionally upper continuous lattice $L$ can be regularly embedded in a complete lattice which satisfies every identity that holds in $L$.

Throughout the usual notation and terminology is used with perhaps the following exceptions. Lattice join, meet, inclusion, and proper inclusion are denoted respectively by the symbols $\cup, \cap$, $<$, while set sum, product, inclusion, and proper inclusion are denoted respectively by $\vee, \wedge, \subseteq, \subset$.

The example is as follows. Let $I$ be an infinite set. Pick three infinite disjoint subsets $a, b, c \subseteq I$ such that

$$
a \vee b \vee c=I \text {. }
$$

Select two sequences $\left\{d_{n}\right\}_{n<\infty}$ and $\left\{e_{n}\right\}_{n<\infty}$ of subsets of $c$ such that

$$
\begin{aligned}
& c \supset d_{1} \supset d_{2} \supset \cdots \supset d_{n} \supset \cdots, \\
& c \supset e_{1} \supset e_{2} \supset \cdots \supset e_{n} \supset \cdots,
\end{aligned}
$$

and such that the following conditions are satisfied:

(i) for every $n=1,2, \cdots$,

$$
c=d_{n} \vee e_{n}
$$

(ii) for every $m=1,2, \ldots$ we have

$$
d_{m} \wedge e_{m} \nsubseteq \bigwedge_{n<\infty} d_{n}, \quad d_{m} \vee e_{m} \Phi \underset{n<\infty}{\bigwedge_{n}}
$$

Define two more sequences $\left\{s_{n}\right\}_{n<\infty}$ and $\left\{t_{n}\right\}_{n<\infty}$ of subsets of $I$ by

$$
s_{n}=a \vee d_{n}, \quad t_{n}=b \vee e_{n} \quad(n=1,2, \cdots)
$$

Then for each $n=1,2, \cdots$ it follows that $s_{n} \wedge\left(t_{n} \vee c\right)=d_{n}$ and $t_{n} \wedge c=e_{n}$.

Let $L_{1}$ be the collection of all the subsets $d_{n}, e_{n}, s_{n}$, and $t_{n}(n=1,2, \cdots)$, all subsets of the form $d_{n} \wedge e_{m}(n, m=1,2, \cdots)$, and the sets $I, a \vee c, b \vee c, c$, and the null set $\varnothing$. Observe that $L_{1}$ is closed under finite set-sums and set-products. Let $L$ be the collection of all subsets of $I$ of the form

$$
x \vee f
$$

where $x \in L_{1}$ and $f$ is a finite subset of $a \bigvee b$. Then $L$ is a distributive lattice under finite set-sums and set-products, and $L$ contains $L_{1}$ as a sublattice. Because of the disjointness of $a, b, c$ and condition (ii), it follows that $\varnothing$ is the only element of $L$ which is contained in $\Lambda_{n<\infty} d_{n}$, and $\varnothing$ is the only element of $L$ which is contained in $\Lambda_{n<\infty} e_{n}$. Hence in $L$ 


$$
\varnothing=\underset{n<\infty}{\text { g.l.b. }} d_{n}=\underset{n<\infty}{\text { g.l.b. }} e_{n} .
$$

Moreover, if $A=\{x \mid x \subseteq a, x$ finite $\}$ and $B=\{x \mid x \subseteq b, x$ finite $\}$, then $I$ is the only element of $L$ which contains every set in $A$ or $B$, and hence in $L$

$$
I=\operatorname{l.u.b.}_{x \in A \vee B} x
$$

Suppose that $K$ is a complete lattice and that $\phi$ is an isomorphic mapping of $L$ into $K$ which preserves all least upper and greatest lower bounds existing in $L$. Set

$$
\alpha=\bigcup_{x \in A} \phi(x), \quad \beta=\bigcup_{x \in B} \phi(x), \quad \gamma=\phi(c) .
$$

Then since $\phi$ preserves least upper bounds in $L$ it follows from (iv) that

$$
\alpha \cup \beta=\alpha \cup \beta \cup \gamma=\phi(I)
$$

Also, since

$$
\begin{gathered}
\beta \cap \gamma \leqq \phi\left(t_{n}\right) \cap \phi(c)=\phi\left(t_{n} \wedge c\right)=\phi\left(e_{n}\right) \\
\alpha \cap(\beta \cup \gamma) \leqq \phi\left(s_{n}\right) \cap\left(\phi\left(t_{n}\right) \cup \phi(c)\right)=\phi\left(s_{n} \wedge\left(t_{n} \vee c\right)\right)=\phi\left(d_{n}\right)
\end{gathered}
$$

for each $n=1,2, \cdots$, we conclude from (iii) that

$$
\begin{gathered}
\beta \cap \gamma \leqq \bigcap_{n<\infty} \phi\left(e_{n}\right)=\phi(\varnothing), \\
\phi(\varnothing) \leqq \alpha \cap \beta \leqq \alpha \cap(\beta \cup \gamma) \leqq \bigcap_{n<\infty} \phi\left(d_{n}\right)=\phi(\varnothing) .
\end{gathered}
$$

Thus $\beta \neq \beta \cup \gamma$, and $\{\alpha, \beta, \beta \cup \gamma, \phi(I), \phi(\varnothing)\}$ is a five-element nonmodular sublattice of $K$. Hence $K$ is nonmodular, and it follows that $L$ cannot be regularly embedded in any complete modular lattice.

Let us now assume that $L$ is an arbitrary conditionally upper continuous lattice. Let $I(L)$ and $K(L)$ denote respectively the lattice of (ordinary) ideals and the lattice of complete ideals of $L$. To distinguish between the operations in $I(L)$ and $K(L)$, meets and joins in $I(L)$ will be denoted by $\bigcap_{I}$ and $\cup_{I}$, while meets and joins in $K(L)$ will be denoted by $\cap_{K}$ and $\cup_{K}$. More generally, if $p\left(z_{1}, \cdots, z_{n}\right)$ is a lattice polynomial on indeterminants $z_{1}, \cdots, z_{n}$ and $A_{1}, \cdots, A_{n}$ are complete ideals of $L$, then $p\left(A_{1}, \cdots, A_{n}\right)_{I}$ will denote the element of $I(L)$ formed by taking meets and joins in $I(L)$, and $p\left(A_{1}, \cdots, A_{n}\right)_{K}$ will denote the element of $K(L)$ formed by taking meets and joins in $K(L)$. 
First note the following: if $A, B \in K(L)$, then

$$
\begin{aligned}
& A \cup_{K} B=\left\{x \mid x=\bigcup S \text { where } S \subseteq A \cup_{I} B\right\}, \\
& A \cap_{K} B=A \cap_{I} B=A \wedge B .
\end{aligned}
$$

For if $Q$ denotes the right side of (1), then it is clear that $A \cup_{K} B \supseteq Q$ $\supseteq A, B$. Suppose $X$ is a subset of $Q$ such that $U X$ exists in $L$ and that $a \in L$. Then for each $x \in X$ there is a subset $S_{x} \subseteq A \cup_{I} B$ such that $x=\bigcup S_{x}$. Let $C$ be the (ordinary) ideal generated by the set-sum of the $S_{x}(x \in X)$. Then $C \subseteq A \cup_{I} B$, and $\cup C=\bigcup_{x} \cup S_{x}=U X$ exists in $L$. Hence since $C$ is a directed subset of $L$ we have

$$
a \cap \bigcup X=a \cap \cup C=\bigcup_{c \in C} a \cap c .
$$

Thus since $\{a \cap c \mid c \in C\} \subseteq C \subseteq A \cup_{I} B$, it follows that $a \cap \cup X \in Q$. Hence $Q$ is a complete ideal, whence $Q=A \cup_{K} B$. Statement (2) is obvious.

Suppose now that $p\left(z_{1}, \cdots, z_{n}\right)$ is a lattice polynomial on indeterminants $z_{1}, \cdots, z_{n}$. Let $A_{1}, \cdots, A_{n}$ be complete ideals of $L$. Then

(3) $p\left(A_{1}, \cdots, A_{n}\right)_{K}=\left\{x \mid x=U S\right.$ where $\left.S \subseteq p\left(A_{1}, \cdots, A_{n}\right)_{I}\right\}$.

The proof of (3) is by induction on the length of $p$. Let $Q$ denote the right side of equation (3). It is easily checked that

$$
p\left(A_{1}, \cdots, A_{n}\right)_{K} \supseteq p\left(A_{1}, \cdots, A_{n}\right)_{I},
$$

whence it follows that $p\left(A_{1}, \cdots, A_{n}\right)_{K} \supseteq Q$. Assume $p=q \cup_{r}$. If $x \in p\left(A_{1}, \cdots, A_{n}\right)_{K}=q\left(A_{1}, \cdots, A_{n}\right)_{K} \cup_{K} r\left(A_{1}, \cdots, A_{n}\right)_{K}$, then it follows by (1) that a subset $S \subseteq q\left(A_{1}, \cdots, A_{n}\right)_{K} \cup_{I} r\left(A_{1}, \cdots, A_{n}\right)_{K}$ exists such that $x=\bigcup S$. Since $S \subseteq q\left(A_{1}, \cdots, A_{n}\right)_{K} \cup_{I} r\left(A_{1}, \cdots, A_{n}\right)_{K}$, it follows that for each $s \in S$ there exist elements $u_{8} \in q\left(A_{1}, \cdots, A_{n}\right)_{K}$ and $v_{s} \in r\left(A_{1}, \cdots, A_{n}\right)_{K}$ such that

$$
s \leqq u_{8} \cup v_{8} .
$$

By the induction assumption, for each $s \in S$ there are subsets $U_{s} \subseteq q\left(A_{1}, \cdots, A_{n}\right)_{I}$ and $V_{s} \subseteq r\left(A_{1}, \cdots, A_{n}\right)_{I}$ such that

$$
u_{s}=U U_{s}, \quad v_{s}=U V_{s} .
$$

Let $C_{s}$ be the ideal generated by the set-sum of $U_{s}$ and $V_{s}$. Then

$$
C_{s} \subseteq q\left(A_{1}, \cdots, A_{n}\right)_{I} \cup_{I} r\left(A_{1}, \cdots, A_{n}\right)_{I}=p\left(A_{1}, \cdots, A_{n}\right)_{I},
$$

and $U C_{8}=u_{8} \cup v_{s}$ for each $s \in S$. Moreover, since each $C_{8}$ is a directed subset of $L$ we have 


$$
s=s \cap\left(u_{s} \cup v_{s}\right)=s \cap \cup C_{s}=\bigcup_{c \in C_{s}} s \cap c
$$

Hence

$$
x=\bigcup S=\bigcup_{s \in S} \bigcup_{c \in C_{s}} s \cap c
$$

and since $\left\{s \cap c \mid s \in S, c \in C_{s}\right\} \subseteq p\left(A_{1}, \cdots, A_{n}\right)_{I}$, we conclude that $x \in Q$. Thus $p\left(A_{1}, \cdots, A_{n}\right)_{K} \subseteq Q$, whence $p\left(A_{1}, \cdots, A_{n}\right)_{K}=Q$. On the other hand, assume $p=q \cap r$. Let $x \in p\left(A_{1}, \cdots, A_{n}\right)_{K}$. Then by (2) we have $x \in q\left(A_{1}, \cdots, A_{n}\right)_{K}$ and $x \in r\left(A_{1}, \cdots, A_{n}\right)_{K}$. By the assumption of the induction there exists subsets $S \subseteq q\left(A_{1}, \cdots, A_{n}\right)_{I}$ and $T \subseteq r\left(A_{1}, \cdots, A_{n}\right)_{I}$ such that $x=U S=U T$. Let $C$ be the ideal generated by $S$ and $D$ be the ideal generated by $T$. Then UC=UD $=\mathrm{U} S=\mathrm{U} T=x$, and since $C$ and $D$ are directed sets,

$$
x=(\cup C) \cap(\cup D)=\bigcup_{c \in C} \bigcup_{d \in D} c \cap d
$$

Thus $x \in Q$ since $\{c \cap d \mid c \in C, d \in D\} \subseteq p\left(A_{1}, \cdots, A_{n}\right)_{I}$, and it follows that $p\left(A_{1}, \cdots, A_{n}\right)_{K} \subseteq Q$. Hence $p\left(A_{1}, \cdots, A_{n}\right)_{K}=Q$ in this case also, and therefore (3) is proved.

We conclude from (3) that every identity which holds in $I(L)$ also holds in $K(L)$. But it is well known that every identity holding in $L$ also holds in $I(L) .{ }^{2}$ Hence $K(L)$ satisfies every identity that $L$ satisfies.

It remains to note that $K(L)$ is upper continuous. If $X_{\lambda}(\lambda \in \Lambda)$ is a chain of complete ideals, then one verifies similarly to the proof of (1) that

$$
\bigcup_{\lambda \in \Lambda} X_{\lambda}=\left\{x \mid x=U S \text { where } S \subseteq \bigvee_{\lambda} X_{\lambda}\right\}
$$

The upper continuity of $K(L)$ is now a simple consequence of this relationship.

\section{REFERENCES}

1. G. Birkhoff, Lattice theory, Amer. Math. Soc. Colloq. Publ. Vol. 25, rev. ed. Amer. Math. Soc., Providence, R. I., 1948.

2. R. P. Dilworth and J. E. McLaughlin, Distributivity in lattices, Duke Math. J. 19 (1952), 683-694.

\section{UNIVERSITY OF WASHINGTON}

Cf., [1, pp. 79-80]. 